\title{
The PRIMA (Proton Imaging) collaboration: Status of the development of a proton Computed Tomography Scanner
}

\author{
M. Bruzzi, M. Brianzi, M. Bucciolini, M. Carpinelli, G. A. P. Cirrone, C. Civinini, G. Cuttone, D. Lo Presti, \\ S. Pallotta, C. Pugliatti, N. Randazzo, F. Romano, V. Sipala, M. Scaringella, C. Stancampiano, C. Talamonti, \\ M. Tesi, E. Vanzi, M. Zani
}

\begin{abstract}
The PRIMA (Proton IMAging) experiment is aimed at developing a proton Computed Tomography (pCT) prototype based on tracking single protons. Approaches to the pCT consist in the use of silicon microstrip detectors to measure the position and direction of individual protons before and after they traverse the phantom, in view to reconstruct the trajectory of each proton, and a calorimeter for measuring particle residual energy. A small prototype was tested, under $62 \mathrm{MeV}$ proton beam at Laboratory Nazionali del Sud, (Catania Italy) and under $180 \mathrm{MeV}$ proton beam at Svedberg Laboratories, Uppsala Universitet, (Uppsala Sweden). Several experiments concerning proton imaging, tomography and radiography, were carried out during these beam tests. Radiographies have been reconstructed with different methods: results show the good performances of the device, with spatial resolution fulfilling the stringent requirements of a pCT device.
\end{abstract}

\footnotetext{
Manuscript received November 16, 2012. This work was supported by INFN CSN5 PRIMA+ experiment.

C. Civinini is with INFN - Florence Division, Florence, Italy (e-mail: civinini@fi.infn.it).

M. Brianzi is with INFN - Florence Division, Florence, Italy.

M. Bruzzi and R. Mori are with Energetics Department, University of Florence and INFN - Florence Division, Florence, Italy. Mara Bruzzi is spokesperson of the experiment.

M. Bucciolini, S. Pallotta, C. Talamonti and Margherita Zani are with Clinical Physiopathology Department, University of Florence and INFN Florence Division, Florence, Italy.

M. Carpinelli is with INFN - Cagliari Division.

G. A. P. Cirrone and G. Cuttone are with INFN - Laboratori Nazionali del Sud, Catania, Italy

D. Lo Presti, C. Pugliatti and C. Stancampiano are with Physics Department, University of Catania and INFN - Catania Division, Catania, Italy.

N. Randazzo is with INFN - Catania Division, Catania, Italy.

F. Romano is with Centro Studi e Ricerche e Museo Storico della Fisica, Rome, Italy and INFN - Laboratori Nazionali del Sud, Catania, Italy.

M. Scaringella and M. Tesi are with Energetics Departments, University of Florence, Italy.

V. Sipala is with Chemistry and Pharmacy Department, University of Sassari, Sassari, Italy and INFN - Cagliari Division.

E. Vanzi is with SOD Fisica Medica, Azienda Ospedaliero-Universitaria Careggi, Florence, Italy.
}

\section{INTRODUCTION}

UE to the well-defined range of protons in material, proton therapy is one of the most precise forms of cancer therapy, allowing for enhancing the sparing of surrounding healthy tissues. Nonetheless, the strong potential of this technique is up to now not fully exploited, being based on X-ray
computed tomography (CT) for the calculation of relative electron density values, a procedure resulting in uncertainties of several $\mathrm{mm}$, due to the different interaction of protons and photons in matter. This problem can be overcome by means of the implementation of a proton CT technique, able to directly measuring the stopping power map using a proton beam with kinetic energy sufficient to cross the patient body [1-5]. This technique is based on the measurement of both the trajectory of each proton both upstream and downstream the target and the residual proton kinetic energy [3-6]. The objective of the PRIMA (PRoton IMAging) project, funded by INFN CSN5, has been to prove feasibility of proton $\mathrm{CT}(\mathrm{pCT})$, by manufacturing and testing a prototype based on single-proton tracking with microstrip silicon detectors and residual energy evaluation with scintillating crystals [7-15].

\section{THE PCT DETECTOR SYSTEM}

In view to get proton-CT images a high energy proton beam must be used, with particles exiting the patient, therefore typical energies are in the range $250-300 \mathrm{MeV}$. The principle of operation of the apparatus we developed is sketched in Fig. 1. It is made by a tracker, to measure proton trajectories, and a calorimeter, to determine their residual energy [6-9].

The tracker is composed of four silicon microstrip $x-y$ planes to measure the proton coordinates in the plane orthogonal to the beam (x-y).

Each plane is formed by two tracker modules rotated by $90^{\circ}$ one respect to the other. 


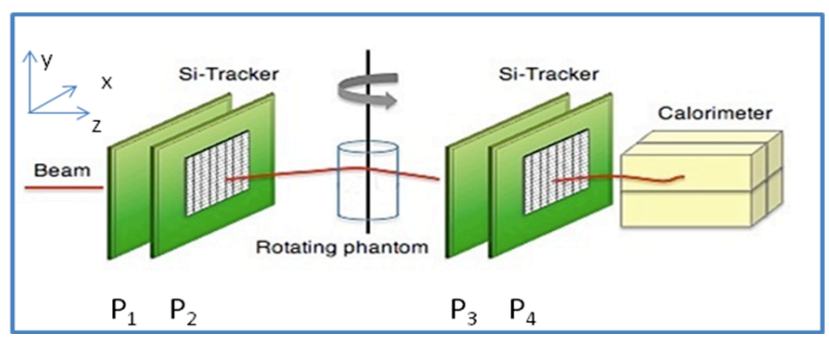

Fig. 1. Sketch of the proton CT system, formed by four $x-y$ planes (p1-p4) and a segmented calorimeter.

Each module includes a front end board, (carrying a 256channels silicon microstrip detectors and 8 ASICs), and a "digital board" (equipped with the FPGA-based digital electronics for data processing, storing and transmission). The detector thickness, $200 \mu \mathrm{m}$, provides a good compromise between low multi-Coulomb scattering, low energy loss in the device and high signal to noise ratio. The ASIC, which contains 32 channels readout in parallel, has been developed in the framework of the INFN PRIMA experiment. To be able to acquire a full image in a fraction of a second, front-end electronics and their data acquisition system should be able to sustain an event rate of $\sim 1 \mathrm{MHz}$. The calorimeter is made of four YAG:Ce optically separated crystals, with size $3 \times 3 \times 10 \mathrm{~cm}^{3}$, arranged as a $2 \times 2$ matrix. This material has been selected due to its short decay time (70 ns) and because its scintillation light is in the photodiode sensitivity range. Photodiodes are simpler to use than photomultiplier and are not sensitive to the magnetic field usually present near the beam line. The $\mathrm{pCT}$ apparatus has been characterized with low $(62 \mathrm{MeV})$ and high $(180 \mathrm{MeV})$ energy beams, respectively at LNS-INFN , Catania, Italy and at Svedberg, Lab, Uppsala, Sweden. A photograph of the PRIMA apparatus at the Svedberg Lab. is shown in Fig. 2.

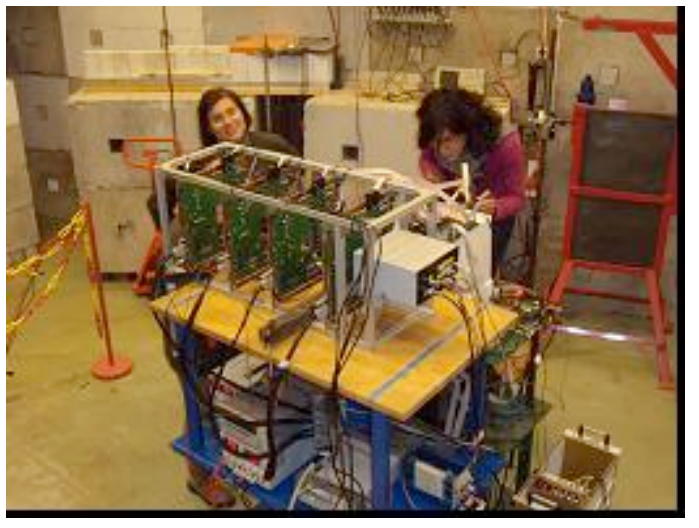

Fig. 2 . Setting-up the PRIMA pCT system at the Svedberg Laboratory beam (180MeV protons).

\section{RADIOGRAPHYC IMAGES}

Customized non-homogeneous phantoms have been prepared for radiography tests. Phantoms have holes of different length within the PolymethylMetacrylate (PMMA) water equivalent material, with thickness opportunely selected in a range of significance for low and high energy proton beam investigations. Figure 3 shows a radiography image obtained by reconstruction of data taken with our apparatus under a $180 \mathrm{MeV}$ proton beam. To reconstruct the image, each event was assigned to a parallel projection ray corresponding to the intersection of the proton trajectory (line connecting the impact points in planes $\mathrm{P}_{2}$ and $\mathrm{P}_{3}$ ) with an ideal plane parallel to the tracker planes and placed at the center of the phantom. This projection plane is then binned in a $256 \times 256$ matrix with a $200 \mu \mathrm{m}$ pixel size [ ]. In case of more than one proton corresponding to the same projection pixel, the mean residual energy was evaluated. To evaluate spatial resolution, profile corresponding to the black line shown in Fig. 5 is reported in Fig. 6. Best fit of the profile is resulting in a standard deviation of $330 \mu \mathrm{m}$. A thorough description of the data analysis process for obtaining radiographyes and on recent results is given in [16].

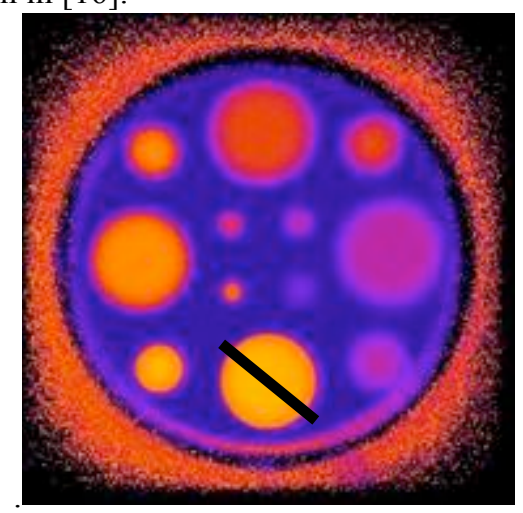

Fig. 3. Radiography reconstructed after $180 \mathrm{MeV}$ proton beam irradiation.

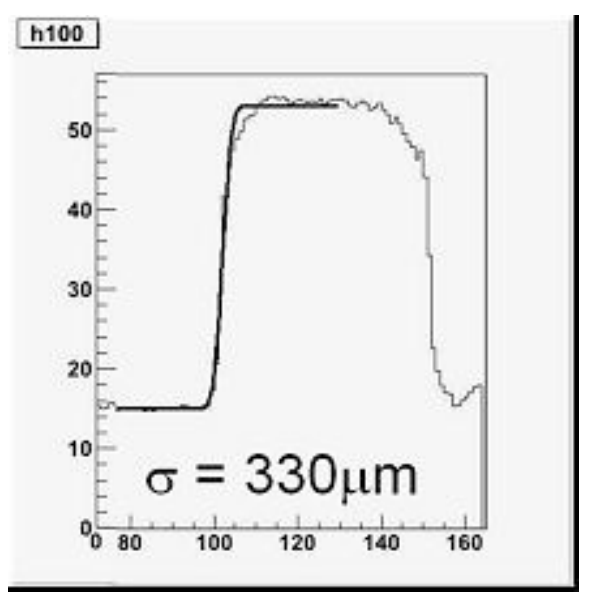

Fig. 4. Profile corresponding to the line in Fig. 3. Best fit resulting in a spatial resolution of $330 \mu \mathrm{m}$ is also shown.

\section{First TOMOGRAPHYC RECONSTRUCTIONS}

To get first tomographic images, we used a PMMA phantom with a diameter of $2 \mathrm{~cm}$ and height $4 \mathrm{~cm}$, equipped with two holes of $4 \mathrm{~mm}$ and $6 \mathrm{~mm}$ diameter respectively and length $2 \mathrm{~cm}$. A photograph of the phantom used on tests at 5 . 
Data have been acquired with 36 different projections (10 degrees steps).

In a conventional X.-ray tomographic analysis, detectors measure the radiation transmitted through the body at different incidence angles, the map of X-ray attenuation coefficient inside the body is then obtained through a tomographic reconstruction of the acquired projections using the Filtered Back Projection (FBP) algorithm, that back-projects the measured data on straight parallel lines perpendicular to the projection direction.

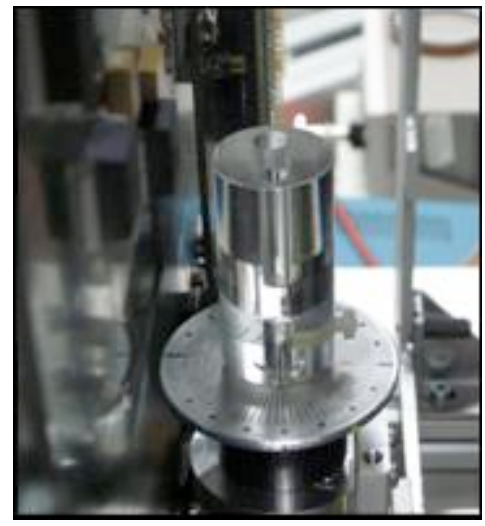

Fig. 5 Photograph of the phantom used for getting first proton computed tomographic images.

This procedure is not suited in principle for pCT image reconstruction, due to the presence of MCS: a description of the proton path must be included in numerical reconstruction methods. The integral imaging equation is then translated into a system of linear equations solved with iterative algorithms such as Algebraic Reconstruction technique (ART). Main disadvantage of this process against FBP is its complexity. In fact, FBP is a simple a fast method of tomographic reconstruction allowing for a first assessment of the object to be imaged, quite precious in view e.g. to get a quick evaluation of the tomographic data quality, to produce a first image as the starting point of an iterative method and for patient positioning verifications. For these reasons we reconstructed our first tomographic images with the FBP algorithm, by calculating and integrating the ratio of the mass stopping power in water as proposed by Wang [17].

Numerical integration was performed starting from NIST stopping power data for water. The coordinates of the porton on P3 plane, binned in a $256 \times 256$ matrix with a 200 um pixel size were considered and, in case of more than one proton corresponding to the same pixel, the mean resisual energy was evaluated. Images have been reconstructed in a $256 \times 256 \times 256$ pixel image volume using the FBP software.

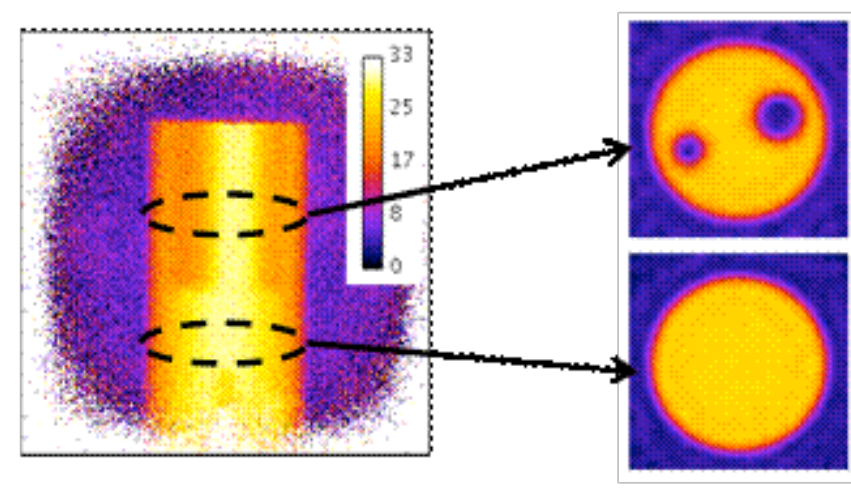

Fig. 6. Tomographic images reconstructed from data taken at the LNSINFN with $62 \mathrm{MeV}$ proton beam, for two different slices of the phantom, in homogeneous and non-homogeneous regions respectively.

A Butterworth filter was used, more details on the reconstruction process are given in [18]. With this technique a volume reconstruction is completed in $22 \mathrm{~s}$, as an example, tomographic images reconstructed from data taken at the LNS-INFN with a $62 \mathrm{MeV}$ proton beam are shown for two different slices of the phantom, in homogeneous and nonhomogeneous regions respectively.

\section{UP-GRADED SYSTEM}

An upgrade of the prototype, using the same detector technologies but with an increased active area $\left(5 \times 20 \mathrm{~cm}^{2}\right)$, higher rate capability and on-line data transfer, is now under development. The choice of the rectangular geometry was made in view of using the instrument to perform imaging of objects with a size of interest for the application (e.g. a human head) by dividing the phantom in slices. To perform a $\mathrm{pCT}$ measurement in a reasonable amount of time, the DAQ system should be able to readout the experimental apparatus at a rate of the order of $1 \mathrm{MHz}$. In fact one single projection radiography to be used to reconstruct a tomography image should be taken with a number of protons of the order of $10^{6}$. This number has to be multiplied by a factor of the order of 36-72 to uniformly cover the entire $360^{\circ}$ ending to a total acquisition time of the order of one minute. A detailed discussion about this new prototype is given in $[19,20]$.

\section{CONCLUSiOnS}

The PRIMA (Proton IMAging) experiment is aimed at developing a proton Computed Tomography ( $\mathrm{pCT}$ ) prototype based on tracking single protons. Approaches to the $\mathrm{pCT}$ consist in the use of silicon microstrip detectors to measure the position and direction of individual protons before and after they traverse the phantom, in view to reconstruct the trajectory of each proton, and a calorimeter for measuring particle residual energy. A small prototype was tested, under $62 \mathrm{MeV}$ proton beam at Laboratory Nazionali del Sud, (Catania Italy) and under $180 \mathrm{MeV}$ proton beam at Svedberg Laboratories, Uppsala Universitet, (Uppsala Sweden). Several experiments concerning proton imaging, tomography and radiography, were carried out during these beam tests. Radiographies have 
been reconstructed with different methods: results show the good performances of the device, with spatial resolution fulfilling the stringent requirements of a pCT device.

An upgraded prototype with larger active area and with improved data acquisition system is now under manufacturing in view to match requirements for pre-clinical tests.

\section{REFERENCES}

[1] A. M. Koehler and V. W. Steward, Science, vol. 179, no. 4076, pp. 913914, 1973.

[2] K. M. Hanson, IEEE Trans. Nucl. Sci., vol. 26, no. 1, p. 1635-1640, 1979

[3] P. Zygmanski, K. P. Gall, M. S. Z. Rabin and S. J. Rosenthal, Phys. Med. Biol. 45 (511) 2000.

[4] R. Schulte et al., IEEE Trans. Nucl. Sci. 51 (866) 2004.

[5] U. Schneider and E. Pedroni, Med. Phys. 21, 1657 (1994)

[6] Mara Bruzzi et al. Prototype tracking studies for proton CT. IEEE Trans. Nucl. Sci., vol. 54, p. 140 - 145 (2007).

[7] G.A.P. Cirrone et al. , The Italian project for a proton imaging device. Nucl.Inst.Meth. A vol. 576, p. 194-197 (2007).

[8] V. Sipala et al. A proton imaging device: Design and status of realization. Nucl.Inst.Meth. A vol. 612, p. 566-570 (2010).

[9] C.Talamonti et al. Proton radiography for clinical applications. Nucl.Inst.Meth. A vol. 612, p. 571-575 (2010).

[10] G.Cirrone et al. Monte Carlo Studies of a Proton Computed Tomography System. IEEE Trans. Nucl. Sci., vol. 54, p. 1487-1491, (2007).

[11] C. Civinini et al., Towards a proton imaging system. Nucl.Inst.Meth. A vol., p. 1-4, (2010).

[12] D.Menichelli et al., Characterization of a silicon strip detector and a $\mathrm{YaG}: \mathrm{Ce}$ calorimeter for a proton computed radiography apparatus. IEEE Trans. Nucl. Sci., vol. Volume: 57 Issue: 1 Part 1, p. 8-16, (2010).

[13] V.Sipala et al., PRIMA: An apparatus for medical application. Nucl.Inst.Meth. A vol. 658, p. 73-77, (2011).

[14] Valeria Sipala at al., Tomographic images by proton Computed Tomography system for proton therapy applications, 2011 IEEE Nuclear Science Symposium Conference Record. p. 3505-3507, ISBN: 9781467301206, doi: 10.1109/NSSMIC.2011.6152644

[15] GAP.Cirrone et al., Monte Carlo evaluation of the Filtered Back Projection method for image reconstruction in proton computed tomography, Nucl.Inst.Meth. A vol. 658, p. 78-83,(2011).

[16] C. Talamonti et al. PRIMA Proton Imaging for Clinical Application, 2012 IEEE Nuclear Science Symposium Conference Record.

[17] Wang

[18] E. Vanzi et al., "The PRIMA collaboration: preliminary results in FBP reconstruction of pCT data", presented at RESMDD12 Conference, Florence, October 9-12, 2012, submitted to Nucl. Instrum. Meth A.

[19] C. Civinini et al. Development of a Proton Computed Tomography scanner, 2012 IEEE Nuclear Science Symposium Conference Record.

[20] M. Scaringella et al., The PRIMA (Proton Imaging) collaboration: development of a proton Computed Tomography apparatus, presented at RESMDD12 Conference, Florence, October 9-12, 2012, submitted to Nucl. Instrum. Meth A. 